\title{
The Spreading Residue Harmonic Balance Method for Strongly Nonlinear Vibrations of a Restrained Cantilever Beam
}

\author{
Y. H. Qian, ${ }^{1}$ J. L. Pan, ${ }^{1}$ S. P. Chen, ${ }^{2}$ and M. H. Yao ${ }^{3}$ \\ ${ }^{1}$ College of Mathematics, Physics and Information Engineering, Zhejiang Normal University, Jinhua, Zhejiang 321004, China \\ ${ }^{2}$ College of Mathematics, Xiamen University of Technology, Xiamen 361024, China \\ ${ }^{3}$ College of Mechanical Engineering, Beijing University of Technology, Beijing 100124, China \\ Correspondence should be addressed to Y. H. Qian; qyh2004@zjnu.cn
}

Received 2 October 2016; Revised 21 February 2017; Accepted 20 March 2017; Published 10 April 2017

Academic Editor: Zhi-Yuan Sun

Copyright (C) 2017 Y. H. Qian et al. This is an open access article distributed under the Creative Commons Attribution License, which permits unrestricted use, distribution, and reproduction in any medium, provided the original work is properly cited.

\begin{abstract}
The exact solutions of the nonlinear vibration systems are extremely complicated to be received, so it is crucial to analyze their approximate solutions. This paper employs the spreading residue harmonic balance method (SRHBM) to derive analytical approximate solutions for the fifth-order nonlinear problem, which corresponds to the strongly nonlinear vibration of an elastically restrained beam with a lumped mass. When the SRHBM is used, the residual terms are added to improve the accuracy of approximate solutions. Illustrative examples are provided along with verifying the accuracy of the present method and are compared with the HAM solutions, the EBM solutions, and exact solutions in tables. At the same time, the phase diagrams and time history curves are drawn by the mathematical software. Through analysis and discussion, the results obtained here demonstrate that the SRHBM is an effective and robust technique for nonlinear dynamical systems. In addition, the SRHBM can be widely applied to a variety of nonlinear dynamic systems.
\end{abstract}

\section{Introduction}

A lot of problems in physical, mechanical, and aeronautical technology and even in structural applications are essentially nonlinear. Majority of the nonlinear dynamical models are mainly composed of a group of differential equations and auxiliary conditions for modeling processes [1]. In general, it is difficult to obtain the exact solution for strongly nonlinear high dimensional dynamic systems. Hence, the analytical approximate solution of the nonlinear problem has become the research object of many scholars in recent years [2-28].

Generally speaking, the fifth-order Duffing type problem with the inertial and static nonlinear terms is sophisticated all the better [29]. Recently, some scholars have tried to study this kind of nonlinear problem. For instance, Telli and Kopmaz [30] and Lai and Lim [31] used the harmonic balance method to study the linear and nonlinear springs. S.-S. Chen and C.-K. Chen [32] dealt with this fifth-order nonlinear problem by applying the differential transformation approach. Subsequently, Ganji et al. [33] and Mehdipour et al. [34], respectively, brought in the homotopy perturbation method, amplitude-frequency formulation, and the energy balance method. They used these methods to solve this strongly nonlinear problem, and lower-order approximate solutions are yielded. Qian et al. [35] studied the nonlinear vibrations of cantilever beam by the HAM. Latterly, Guo et al. $[36,37]$ have presented the residue harmonic balance solution procedure to approximate the periodic behavior of different oscillation systems and they have obtained some more accurate results. Ju and Xue $[38,39]$ proposed the global residue harmonic balance method to study strongly nonlinear systems. Comparing the obtained solutions with the exact one, they discovered that the approximate results excellently agree with the exact one. Lee [40] used the multilevel residue harmonic balance method to solve a nonlinear panel coupled with extended cavity.

The principal intention of this paper is to investigate the utility of the spreading residue harmonic balance method (SRHBM) [36] for the fifth-order strongly nonlinear problem. The paper consists of the following several parts. Section 2 describes how the strongly nonlinear equation is educed from the governing equations of the cantilever beam model in 


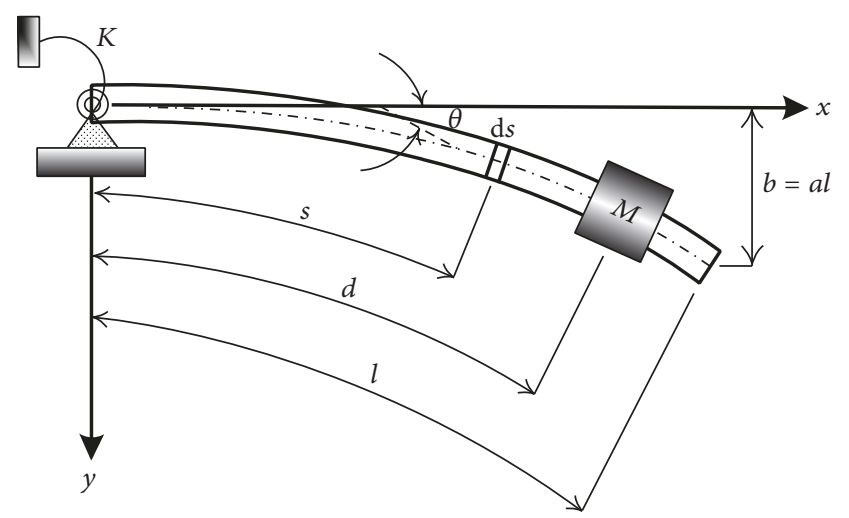

FIGURE 1: Geometry and coordinate system for a beam with a lumped mass.

a nutshell. In Section 3, the SRHBM is introduced and the solution process of different order solutions will be presented. The numerical examples of the SRHBM are rendered and compared with other solutions in Section 4. Finally, conclusion of the paper is drawn in Section 5.

\section{Mathematical Formulation}

An isotropic slender beam with uniform length $l$ and mass $m$ per unit length is considered, as shown in Figure 1 [25]. It is assumed that the beam thickness is much smaller than the beam length, so the effects of shear deformation and rotary inertia can be ignored. The angle of inclination is $\theta$ and the beam displacement is $a=b / l$. For the boundary condition constraints, one of the conditions is hinged at the bottom of a rotational spring with stiffness $K$, and the other condition is independent. Moreover, the intermediate lumped mass $M$ is also connected in $s=d$ along the beam span. By the Euler-Lagrange differential equation, the fifthorder Duffing type temporal problem with strongly inertial and static nonlinearities is able to be derived as follows [25]:

$$
\begin{aligned}
& \ddot{x}+x+\varepsilon_{1} x^{2} \ddot{x}+\varepsilon_{1} x \dot{x}^{2}+\varepsilon_{2} x^{4} \ddot{x}+2 \varepsilon_{2} x^{3} \dot{x}^{2}+\varepsilon_{3} x^{3} \\
& \quad+\varepsilon_{4} x^{5}=0 \\
& x(0)=A \\
& \dot{x}(0)=0
\end{aligned}
$$

where $x$ is the dimensionless deflection at the tip of the beam, $A$ is the maximum amplitude, the overdot indicates the derivative relative to $t$, and $\varepsilon_{1}, \varepsilon_{2}, \varepsilon_{3}$, and $\varepsilon_{4}$ are parameters. For the complete formulation of (1), readers are referred to [25] for details.

\section{Solution Methodology}

In the following, the spreading residue harmonic is used to solve (1). Firstly, by introducing a new variable $\tau=\omega t$ and substituting it into (1), we can get

$$
\begin{aligned}
& \omega^{2} x^{\prime \prime}+x+\varepsilon_{1} \omega^{2} x^{2} x^{\prime \prime}+\varepsilon_{1} \omega^{2} x x^{\prime 2}+\varepsilon_{2} \omega^{2} x^{4} x^{\prime \prime} \\
& \quad+2 \varepsilon_{2} \omega^{2} x^{3} x^{\prime 2}+\varepsilon_{3} x^{3}+\varepsilon_{4} x^{5}=0,
\end{aligned}
$$

$$
\begin{aligned}
& x(0)=A, \\
& x^{\prime}(0)=0,
\end{aligned}
$$

where $x^{\prime}$ represents the first-order derivative with respect to $\tau$ and $\omega$ is the unknown angular frequency of (1).

Since we discuss the existence of a periodic solution, we usually choose the base functions

$$
\{\cos [(2 k-1) \tau] \mid k=1,2,3, \ldots\}
$$

and we find the expression of the steady state solutions

$$
\begin{aligned}
x(\tau) & =x_{0}(\tau)+p x_{1}(\tau)+p^{2} x_{2}(\tau)+\cdots \\
\omega^{2} & =\omega_{0}^{2}+p \omega_{1}+p^{2} \omega_{2}+\cdots
\end{aligned}
$$

where $p$ is an order parameter and $\omega_{i}(i=0,1,2, \ldots)$ are unknown.

Next, we mainly analyze the zeroth-order harmonic approximation, the first-order harmonic approximation, and the second-order harmonic approximation.

3.1. The Zeroth-Order Harmonic Approximation. To meet the initial conditions in (2), we can set the following initial guess solution of $x(\tau)$; that is,

$$
x_{0}(\tau)=A \cos (\tau), \quad \tau=\omega_{0} t
$$

Substituting (5) into (2), the equation is yielded:

$$
\begin{aligned}
& R_{0}(\tau)=\omega_{0}^{2} x_{0}^{\prime \prime}(\tau)+x_{0}(\tau)+\varepsilon_{1} \omega_{0}^{2} x_{0}^{2}(\tau) x_{0}^{\prime \prime}(\tau) \\
& +\varepsilon_{1} \omega_{0}^{2} x_{0}(\tau) x_{0}^{\prime 2}(\tau)+\varepsilon_{2} \omega_{0}^{2} x_{0}^{4}(\tau) x_{0}^{\prime \prime}(\tau) \\
& +2 \varepsilon_{2} \omega_{0}^{2} x_{0}^{3}(\tau) x_{0}^{\prime 2}(\tau)+\varepsilon_{3} x_{0}^{3}(\tau)+\varepsilon_{4} x_{0}^{5}(\tau)=[A(1 \\
& \left.\left.-\omega_{0}^{2}+\frac{3}{4} A^{2} \varepsilon_{3}-\frac{1}{2} \varepsilon_{1} A^{2} \omega_{0}^{2}+\frac{5}{8} A^{4} \varepsilon_{4}-\frac{3}{8} A^{4} \varepsilon_{2} \omega_{0}^{2}\right)\right] \\
& \cdot \cos (\tau)+\left[A ^ { 3 } \left(\frac{1}{4} \varepsilon_{3}-\frac{1}{2} \varepsilon_{1} \omega_{0}^{2}+\frac{5}{16} A^{2} \varepsilon_{4}\right.\right. \\
& \left.\left.-\frac{7}{16} A^{2} \varepsilon_{2} \omega_{0}^{2}\right)\right] \cos (3 \tau)+\left[\frac{1}{16} A^{5}\left(\varepsilon_{4}-3 \varepsilon_{2} \omega_{0}^{2}\right)\right] \\
& \cdot \cos (5 \tau) .
\end{aligned}
$$

$R_{0}(\tau)$ denotes the zeroth-order residual term. When $R_{0}(\tau)=0, x_{0}(\tau)$ is the exact solution.

Based on the Galerkin procedure, the secular term $\cos (\tau)$ cannot appear on the right hand side of (6). Equating the term's coefficient to zero, we obtain a linear equation containing an unknown $\omega_{0}$. Through solving that equation, we are able to work out the unknown frequency $\omega_{0}$ :

$$
\omega_{0}=\sqrt{\frac{8+6 A^{2} \varepsilon_{3}+5 A^{4} \varepsilon_{4}}{8+4 A^{2} \varepsilon_{1}+3 A^{4} \varepsilon_{2}}} .
$$


In addition, the zeroth-order approximation solution can be obtained as follows:

$$
x_{0}(\tau)=A \cos \left(\omega_{0} t\right) .
$$

When the obtained zeroth-order solution is substituted into (6), the terms of $\cos (3 \tau)$ and $\cos (5 \tau)$ generally are not zero.

3.2. The First-Order Harmonic Approximations. Substituting (4) into (2), the coefficient of parameter $p$ is put forward, and we can obtain

$$
\begin{aligned}
& \phi_{1}(\tau)=\omega_{0}^{2}\left[x_{1}^{\prime \prime}\right. \\
& +\varepsilon_{1}\left(x_{1} x_{0}^{\prime 2}+2 x_{0} x_{0}^{\prime} x_{1}^{\prime}+2 x_{0} x_{1} x_{0}^{\prime \prime}+x_{0}^{2} x_{1}^{\prime \prime}\right) \\
& \left.+\varepsilon_{2}\left(6 x_{0}^{2} x_{1} x_{0}^{\prime 2}+4 x_{0}^{3} x_{0}^{\prime} x_{1}^{\prime}+4 x_{0}^{3} x_{1} x_{0}^{\prime \prime}+x_{0}^{4} x_{1}^{\prime \prime}\right)\right] \\
& +\omega_{1}\left[x_{0}^{\prime \prime}+\varepsilon_{1}\left(x_{0} x_{0}^{\prime 2}+x_{0}^{2} x_{0}^{\prime \prime}\right)\right. \\
& \left.+\varepsilon_{2}\left(2 x_{0}^{3} x_{0}^{\prime 2}+x_{0}^{4} x_{0}^{\prime \prime}\right)\right]+x_{1}+3 \varepsilon_{3} x_{0}^{2} x_{1}+5 \varepsilon_{4} x_{0}^{4} x_{1} .
\end{aligned}
$$

According to (3), we choose the following equation as the solution of the representation:

$$
x_{1}(\tau)=a_{3,1}[\cos (\tau)-\cos (3 \tau)],
$$

where $a_{3,1}$ is the unknown.

Combining (10) with (9), we consider

$$
R_{1}(\tau)=\phi_{1}(\tau)+R_{0}(\tau) .
$$

We can obtain

$$
\begin{aligned}
R_{1}(\tau) & =\left[a_{3,1}\left(1-\omega_{0}^{2}\right)-A \omega_{1}+\frac{3}{2} A^{2} a_{3,1} \varepsilon_{3}\right. \\
& \left.-\frac{1}{2} A^{3} \omega_{1} \varepsilon_{1}+\frac{5}{16} A^{4} a_{3,1}\left(5 \varepsilon_{4}+\varepsilon_{2} \omega_{0}^{2}\right)-\frac{3}{8} A^{5} \varepsilon_{2} \omega_{1}\right] \\
& \cdot \cos (\tau)+\left[\left(9 a_{3,1} \omega_{0}^{2}-a_{3,1}\right)\right. \\
+ & A^{2}\left(\frac{7}{2} a_{3,1} \varepsilon_{1} \omega_{0}^{2}-\frac{3}{4} a_{3,1} \varepsilon_{3}\right) \\
+ & A^{3}\left(\frac{1}{4} \varepsilon_{3}-\frac{1}{2} \varepsilon_{1}\left(\omega_{0}^{2}+\omega_{1}\right)\right) \\
+ & A^{4}\left(\frac{31}{16} a_{3,1} \omega_{0}^{2} \varepsilon_{2}-\frac{5}{16} a_{3,1} \varepsilon_{4}\right) \\
+ & \left.A^{5}\left(\frac{5}{16} \varepsilon_{4}-\frac{7}{16} \varepsilon_{2}\left(\omega_{0}^{2}+\omega_{1}\right)\right)\right] \cos (3 \tau) \\
+ & {\left[A^{2}\left(\frac{9}{2} a_{3,1} \varepsilon_{1} \omega_{0}^{2}-\frac{3}{4} a_{3,1} \varepsilon_{3}\right)\right.} \\
+ & A^{4} a_{3,1}\left(\frac{61}{16} \varepsilon_{2} \omega_{0}^{2}-\frac{15}{16} \varepsilon_{4}\right) \\
+ & A^{5}\left(\frac{1}{16} \varepsilon_{4}-\frac{3}{16} \varepsilon_{2}\left(\omega_{0}^{2}+\omega_{3,1}\left(\frac{31}{16} \varepsilon_{2} \omega_{0}^{2}-\frac{5}{16} \varepsilon_{4}\right)\right] \cos (7 \tau)\right. \\
+ & \cos (5 \tau)
\end{aligned}
$$

To increase the accuracy, (11) should be added in the zerothorder residual term $R_{0}(\tau)$.

Based on the Galerkin procedure, (12) should not contain secular terms. Letting coefficients of $\cos (\tau)$ and $\cos (3 \tau)$ be zeros, hence, we obtain a linear equation set containing two unknowns $\omega_{1}$ and $a_{3,1}$. Through solving the equation set, we can get

$$
\begin{aligned}
& \omega_{1} \\
& =\frac{A^{2}\left(16+24 A^{2} \varepsilon_{3}+25 A^{4} \varepsilon_{4}+\left(5 A^{4} \varepsilon_{2}-16\right) \omega_{0}^{2}\right) N}{M}, \\
& a_{3,1}=\frac{2 A^{3}\left(8+4 A^{2} \varepsilon_{1}+3 A^{4} \varepsilon_{2}\right) N}{M},
\end{aligned}
$$

where

$$
\begin{aligned}
M & =256\left(1-9 \omega_{0}^{2}\right)+A^{8} \varepsilon_{2}\left(205 \varepsilon_{4}-151 \varepsilon_{2} \omega_{0}^{2}\right) \\
& +16 A^{6}\left(15 \varepsilon_{2} \varepsilon_{3}+15 \varepsilon_{1} \varepsilon_{4}-34 \varepsilon_{1} \varepsilon_{2} \omega_{0}^{2}\right) \\
& -16 A^{4}\left(-13 \varepsilon_{2}-18 \varepsilon_{1} \varepsilon_{3}-5 \varepsilon_{4}\right. \\
& \left.+4\left(7 \varepsilon_{1}^{2}+23 \varepsilon_{2}\right) \omega_{0}^{2}\right)+64 A^{2}\left(3 \varepsilon_{3}+\varepsilon_{1}\left(4-34 \omega_{0}^{2}\right)\right), \\
N & =4 \varepsilon_{3}-8 \varepsilon_{1} \omega_{0}^{2}+A^{2}\left(5 \varepsilon_{4}-7 \varepsilon_{2} \omega_{0}^{2}\right) .
\end{aligned}
$$

Therefore, the first-order harmonic approximation can be procured:

$$
\begin{aligned}
\omega_{(1)} & =\sqrt{\omega_{0}^{2}+\omega_{1}}, \\
x_{(1)}(\tau) & =x_{(0)}(\tau)+x_{1}(\tau) \\
& =\left(A+a_{3,1}\right) \cos (\tau)-a_{3,1} \cos (3 \tau), \\
& \tau=\omega_{(1)} t .
\end{aligned}
$$

When the obtained first-order solution is substituted into (12), the terms of $\cos (5 \tau)$ and $\cos (7 \tau)$ commonly are not zero.

3.3. The Second-Order Harmonic Approximations. Substituting (4) to (2) and presenting the coefficient of parameter $p^{2}$ yield

$$
\begin{aligned}
& \phi_{2}(\tau)=\omega_{0}^{2}\left(6 \varepsilon_{2} x_{0} x_{1}^{2} x_{0}^{\prime 2}+\varepsilon_{1} x_{2} x_{0}^{\prime 2}+6 \varepsilon_{2} x_{2} x_{0}^{2} x_{0}^{\prime 2}\right. \\
& +2 \varepsilon_{1} x_{1} x_{0}^{\prime} x_{1}^{\prime}+12 \varepsilon_{2} x_{0}^{2} x_{1} x_{0}^{\prime} x_{1}^{\prime}+\varepsilon_{1} x_{0} x_{1}^{2}+2 \varepsilon_{2} x_{0}^{3} x_{1}^{\prime 2} \\
& +2 \varepsilon_{1} x_{0} x_{0}^{\prime} x_{2}^{\prime}+4 \varepsilon_{2} x_{0}^{3} x_{0}^{\prime} x_{2}^{\prime}+\varepsilon_{1} x_{1}^{2} x_{0}^{\prime \prime}+6 \varepsilon_{2} x_{0}^{2} x_{1}^{2} x_{0}^{\prime \prime} \\
& +2 \varepsilon_{1} x_{0} x_{2} x_{0}^{\prime \prime}+4 \varepsilon_{2} x_{0}^{3} x_{2} x_{0}^{\prime \prime}+2 \varepsilon_{1} x_{0} x_{1} x_{1}^{\prime \prime} \\
& \left.+4 \varepsilon_{2} x_{0}^{3} x_{1} x_{1}^{\prime \prime}+x_{2}^{\prime \prime}+\varepsilon_{1} x_{0}^{2} x_{2}^{\prime \prime}+\varepsilon_{2} x_{0}^{4} x_{2}^{\prime \prime}\right) \\
& +\omega_{1}\left(\varepsilon_{1} x_{1} x_{0}^{\prime 2}+6 \varepsilon_{2} x_{0}^{2} x_{1} x_{0}^{\prime 2}+2 \varepsilon_{1} x_{0} x_{0}^{\prime} x_{1}^{\prime}\right. \\
& +4 \varepsilon_{2} x_{0}^{3} x_{0}^{\prime} x_{1}^{\prime}+2 \varepsilon_{1} x_{0} x_{1} x_{0}^{\prime \prime}+4 \varepsilon_{2} x_{0}^{3} x_{1} x_{0}^{\prime \prime}+x_{1}^{\prime \prime}
\end{aligned}
$$




$$
\begin{aligned}
& \left.+\varepsilon_{1} x_{0}^{2} x_{1}^{\prime \prime}+\varepsilon_{2} x_{0}^{4} x_{1}^{\prime \prime}\right)+\omega_{2}\left(\varepsilon_{1} x_{0} x_{0}^{\prime 2}+2 \varepsilon_{2} x_{0}^{3} x_{0}^{\prime 2}\right. \\
& \left.+x_{0}^{\prime \prime}+\varepsilon_{1} x_{0}^{2} x_{0}^{\prime \prime}+\varepsilon_{2} x_{0}^{4} x_{0}^{\prime \prime}\right)+3 \varepsilon_{3} x_{0} x_{1}^{2}+10 \varepsilon_{4} x_{0}^{3} x_{1}^{2} \\
& +x_{2}+3 \varepsilon_{3} x_{0}^{2} x_{2}+5 \varepsilon_{4} x_{0}^{4} x_{2} .
\end{aligned}
$$

Observing (16), we know it is linear with respect to $\omega_{2}$ and $x_{2}(\tau)$. In accordance with the form of (3), we apply

$$
\begin{aligned}
x_{2}(\tau)= & a_{3,2}[\cos (\tau)-\cos (3 \tau)] \\
& +a_{5,2}[\cos (\tau)-\cos (5 \tau)],
\end{aligned}
$$

where $a_{3,2}$ and $a_{5,2}$ are unknowns.

Combining (17) with (16) and calculating the equation

$$
R_{2}(\tau)=\phi_{2}(\tau)+R_{1}(\tau)
$$

we can obtain

$$
\begin{aligned}
& R_{2}(\tau)=\left[A\left(\frac{9}{4} a_{3,1}^{2} \varepsilon_{3}-\frac{7}{2} a_{3,1}^{2} \varepsilon_{1} \omega_{0}^{2}-\omega_{2}\right)\right. \\
& +A^{2}\left(\frac{3}{2} a_{3,2} \varepsilon_{3}+\frac{9}{4} a_{5,2} \varepsilon_{3}-\frac{3}{2} a_{5,2} \varepsilon_{1} \omega_{0}^{2}\right) \\
& +A^{3}\left(\frac{15}{4} a_{3,1}^{2} \varepsilon_{4}-\frac{13}{4} a_{3,1}^{2} \varepsilon_{2} \omega_{0}^{2}-\frac{1}{2} \varepsilon_{1} \omega_{2}\right)+\frac{1}{16} \\
& \cdot A^{4}\left(25 a_{3,2} \varepsilon_{4}+45 a_{5,2} \varepsilon_{4}+5 a_{3,2} \varepsilon_{2} \omega_{0}^{2}-15 a_{5,2} \varepsilon_{2} \omega_{0}^{2}\right. \\
& \left.+5 a_{3,1} \varepsilon_{2} \omega_{1}\right)-\frac{3}{8} A^{5} \varepsilon_{2} \omega_{2}+\left(a_{3,2}+a_{5,2}-a_{3,2} \omega_{0}^{2}\right. \\
& \left.\left.-a_{5,2} \omega_{0}^{2}-a_{3,1} \omega_{1}\right)\right] \cos (\tau)+\left[A a _ { 3 , 1 } ^ { 2 } \left(\frac{17}{2} \varepsilon_{1} \omega_{0}^{2}\right.\right. \\
& \left.-\frac{9}{4} \varepsilon_{3}\right)+A^{2}\left(\frac{7}{2} a_{3,2} \varepsilon_{1} \omega_{0}^{2}-\frac{3}{4} a_{3,2} \varepsilon_{3}\right. \\
& \left.+\varepsilon_{1}\left(3 a_{5,2} \omega_{0}^{2}+\frac{7}{2} a_{3,1} \omega_{1}\right)\right)-\frac{1}{2} \\
& \cdot A^{3}\left(a_{3,1}^{2}\left(5 \varepsilon_{4}-13 \varepsilon_{2} \omega_{0}^{2}\right)+\varepsilon_{1} \omega_{2}\right)+\frac{1}{16} A^{4}\left(5 a_{5,2} \varepsilon_{4}\right. \\
& \left.-5 a_{3,2} \varepsilon_{4}+31 a_{3,2} \varepsilon_{2} \omega_{0}^{2}+41 a_{5,2} \varepsilon_{2} \omega_{0}^{2}+31 a_{3,1} \varepsilon_{2} \omega_{1}\right) \\
& \left.-\frac{7}{16} A^{5} \varepsilon_{2} \omega_{2}+\left(9 a_{3,2} \omega_{0}^{2}+9 a_{3,1} \omega_{1}-a_{3,2}\right)\right] \cos (3 \tau) \\
& +\left[A a_{31}^{2}\left(\frac{7}{2} \varepsilon_{1} \omega_{0}^{2}-\frac{3}{4} \varepsilon_{3}\right)+A^{2}\left(13 a_{5,2} \varepsilon_{1} \omega_{0}^{2}\right.\right. \\
& -\frac{3}{2} a_{5,2} \varepsilon_{3}-\frac{3}{4}\left(a_{3,1}+a_{3,2}\right)\left(\varepsilon_{3}-6 \varepsilon_{1} \omega_{0}^{2}\right) \\
& \left.+\frac{9}{2} a_{3,1} \varepsilon_{1} \omega_{1}\right)+\frac{1}{2} A^{3} a_{3,1}^{2}\left(17 \varepsilon_{2} \omega_{0}^{2}-5 \varepsilon_{4}\right)+\frac{1}{16} \\
& \cdot A^{4}\left(147 a_{5,2} \varepsilon_{2} \omega_{0}^{2}-25 a_{5,2} \varepsilon_{4}-\left(a_{3,1}+a_{3,2}\right)\left(15 \varepsilon_{4}\right.\right.
\end{aligned}
$$

$$
\begin{aligned}
& \left.\left.-61 \varepsilon_{2} \omega_{0}^{2}\right)+61 a_{3,1} \varepsilon_{2} \omega_{1}\right)+\frac{1}{16} A^{5}\left(\varepsilon_{4}-3 \varepsilon_{2}\left(\omega_{0}^{2}\right.\right. \\
& \left.\left.\left.+\omega_{1}+\omega_{2}\right)\right)+\left(25 a_{5,2} \omega_{0}^{2}-a_{5,2}\right)\right] \cos (5 \tau) \\
& +\left[A\left(\frac{3}{4} a_{3,1}^{2} \varepsilon_{3}-\frac{17}{2} a_{3,1}^{2} \varepsilon_{1} \omega_{0}^{2}\right)-A^{2} a_{5,2}\left(\frac{3}{4} \varepsilon_{3}\right.\right. \\
& \left.-\frac{19}{2} \varepsilon_{1} \omega_{0}^{2}\right)+\frac{1}{8} A^{3} a_{3,1}^{2}\left(5 \varepsilon_{4}-43 \varepsilon_{2} \omega_{0}^{2}\right) \\
& +A^{4}\left(-\frac{5}{16} a_{3,2} \varepsilon_{4}-\frac{5}{4} a_{5,2} \varepsilon_{4}+\frac{31}{16} a_{3,2} \varepsilon_{2} \omega_{0}^{2}\right. \\
& +\frac{39}{4} a_{5,2} \varepsilon_{2} \omega_{0}^{2}-\frac{5}{16} a_{3,2} \varepsilon_{4} \\
& \left.\left.+\frac{1}{16} a_{3,1}\left(31 \varepsilon_{2}\left(\omega_{0}^{2}+\omega_{1}\right)\right)\right)\right] \cos (7 \tau)+\left[\frac{1}{8}\right. \\
& \cdot A^{3} a_{3,1}^{2}\left(5 \varepsilon_{4}-51 \varepsilon_{2} \omega_{0}^{2}\right)-\frac{5}{16} A^{4} a_{5,2}\left(\varepsilon_{4}\right. \\
& \left.\left.-11 \varepsilon_{2} \omega_{0}^{2}\right)\right] \cos (9 \tau) .
\end{aligned}
$$

With the purpose of increasing the accuracy, (18) should be put on the first-order residual term $R_{1}(\tau)$.

In order to prevent the right hand side of (19) from exhibiting the secular terms $\cos (\tau), \cos (3 \tau)$, and $\cos (5 \tau)$, we make all their coefficients equal to zero. Then, we can get three linear equations containing three unknown parameters $a_{3,2}$, $a_{5,2}$, and $\omega_{2}$. According to the three linear equations, we can solve the three unknowns. Thus, the second-order harmonic approximation is shown:

$$
\begin{aligned}
\omega_{(2)} & =\sqrt{\omega_{0}^{2}+\omega_{1}+\omega_{2}}, \\
x_{(2)}(\tau) & =x_{(0)}(\tau)+x_{1}(\tau)+x_{2}(\tau), \quad \tau=\omega_{(2)} t .
\end{aligned}
$$

In conclusion, we draw the $k$ th-order harmonic approximation $(k=2,3,4, \ldots)$

$$
\begin{aligned}
x_{(k)}(\tau) & =x_{(k-1)}(\tau)+x_{k}(\tau), \\
\omega_{(k)} & =\sqrt{\omega_{(k-1)}^{2}+\omega_{k}} \quad k=2,3,4, \ldots, \\
x_{(k-1)}(\tau) & =x_{(k-2)}(\tau)+x_{k-1}(\tau), \\
\omega_{(k-1)} & =\sqrt{\omega_{(k-2)}^{2}+\omega_{k-1}}, \\
x_{k}(\tau) & =\sum_{i=1}^{k} a_{2 i+1, k}[\cos (\tau)-\cos ((2 i+1) \tau)], \\
& x_{(0)}=A \cos (\tau), \omega_{(0)}=\omega_{0}, k=2,3,4, \ldots
\end{aligned}
$$

\section{Results and Discussion}

In order to make sure of the effectiveness of the current technique, we compare the results from the second-order spreading residue harmonic balance approach $\omega_{\text {SRHB }}$ with the 
TABLE 1: Comparison of the SRHBM frequencies, EBM frequencies, HAM frequencies, and the exact frequencies for various parameters.

\begin{tabular}{|c|c|c|c|c|c|c|c|c|c|}
\hline Mode & $A$ & $\varepsilon_{1}$ & $\varepsilon_{2}$ & $\varepsilon_{3}$ & $\varepsilon_{4}$ & $\omega_{\mathrm{EBM}}$ & $\omega_{\mathrm{HAM}}$ & $\omega_{\mathrm{SRHB}}$ & $\omega_{\mathrm{ex}}$ \\
\hline 1 & 1.0 & 0.326845 & 0.129579 & 0.232598 & 0.087584 & 1.01235 & 1.01232 & 1.01004 & 1.01015 \\
\hline 2 & 0.5 & 1.642033 & 0.913055 & 0.313561 & 0.204297 & 0.935046 & 0.938636 & 0.935072 & 0.93639 \\
\hline 3 & 0.2 & 4.051486 & 1.665232 & 0.281418 & 0.149677 & 0.965613 & 0.966516 & 0.965843 & 0.96664 \\
\hline 4 & 0.3 & 8.205578 & 3.145368 & 0.272313 & 0.133708 & 0.860678 & 0.871382 & 0.863939 & 0.86426 \\
\hline
\end{tabular}

TABLE 2: Comparison of the SRHBM relative error, EBM relative error, and HAM relative error with the exact frequencies for various parameters.

\begin{tabular}{cccccccc|cc}
\hline Mode & $A$ & $\varepsilon_{1}$ & $\varepsilon_{2}$ & $\varepsilon_{3}$ & $\varepsilon_{4}$ & $\frac{\left|\omega_{\mathrm{EBM}}-\omega_{\mathrm{ex}}\right|}{\omega_{\mathrm{ex}}} \times 100 \%$ & $\frac{\left|\omega_{\mathrm{HAM}}-\omega_{\mathrm{ex}}\right|}{\omega_{\mathrm{ex}}} \times 100 \%$ & $\frac{\left|\omega_{\mathrm{SRHB}}-\omega_{\mathrm{ex}}\right|}{\omega_{\mathrm{ex}}} \times 100 \%$ \\
\hline 1 & 1.0 & 0.326845 & 0.129579 & 0.232598 & 0.087584 & 0.21789 & 0.21482 & 0.010889 \\
2 & 0.5 & 1.642033 & 0.913055 & 0.313561 & 0.204297 & 0.14353 & 0.23986 & 0.140753 \\
3 & 0.2 & 4.051486 & 1.665232 & 0.281418 & 0.149677 & 0.106244 & 0.01283 & 0.08245 \\
4 & 0.3 & 8.205578 & 3.145368 & 0.272313 & 0.133708 & 0.414459 & 0.82406 & 0.037142 \\
\hline
\end{tabular}
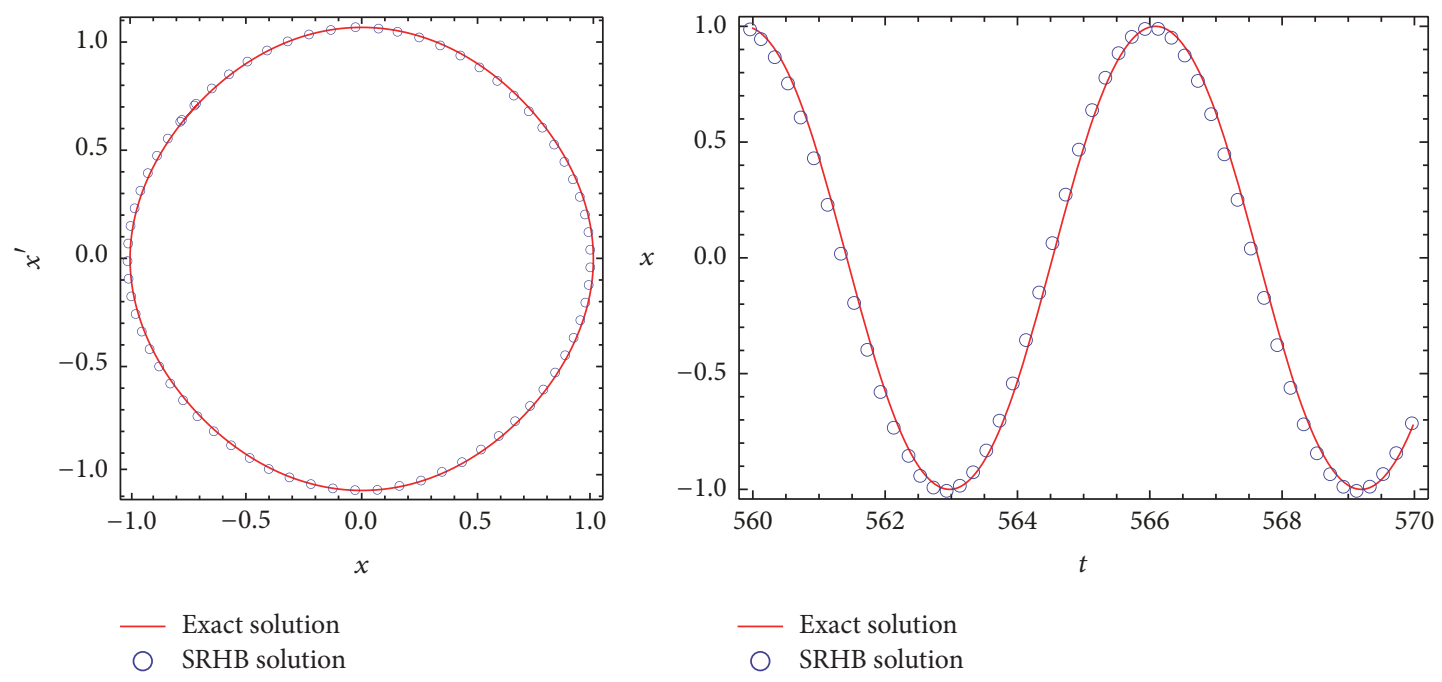

FIGURE 2: Comparison of the approximate and exact solutions of Mode 1.

energy balance method $\omega_{\mathrm{EBM}}[34]$, the homotopy analysis method $\omega_{\text {HAM }}$ [35], and the exact solution $\omega_{\text {ex }}$ [33], which are presented in Table 1 , for different parameters $\varepsilon_{i}(i=1,2,3,4)$ and amplitudes of vibration $A$, where the exact solution $\omega_{\text {ex }}$ is computed using the numerical technique. The relative errors of vibration frequency are tabulated in Table 2.

For Mode 1 in Table 2, we observe that the relative error between $\omega_{\text {SRHB }}$ and $\omega_{\text {ex }}$ is much less than the relative error between $\omega_{\text {HAM }}$ and $\omega_{\text {ex }}$. The same goes for Mode 2 and Mode 4. However, the relative error between $\omega_{\mathrm{SRHB}}$ and $\omega_{\mathrm{ex}}$ for Mode 3 is more than the relative error between $\omega_{\mathrm{HAM}}$ and $\omega_{\mathrm{ex}}$. Hence, we conclude that the accuracy of the second-order SRHBM solutions is improved in Mode 1, Mode 2, and Mode 4. Similarly, from Table 2, the SRHBM relative error is smaller than EBM relative error in Mode 1, Mode 2, Mode 3, and Mode 4. These results show that the approximate solutions obtained by the SRHBM are closer to the exact solutions.

To further demonstrate the accuracy of the spreading residue harmonic balance approach, the time history responses and the phase portrait are rendered for four different sets of parameters in Figures 2-5. From the phase portrait diagram, we obviously discover that the secondorder residue harmonic balance solutions are very consistent with the exact solutions. From the phase portrait, we observe that the system is a periodic motion. Moreover, in the whole range, the presented approximate solutions converge to the exact solutions. Extraordinarily, we argue for $t$ in $[560,570]$.

\section{Conclusions}

In this paper, the spreading residue harmonic balance method is applied to discuss the strongly nonlinear vibration system. Particularly, we take a restrained cantilever beam as an example. The SRHBM does not need to add small parameters in the calculation process. Besides, this approach approximates the exact solution quickly and only the first- or second-order approximations. And by comparing its results with HAM and EBM for various parameters and amplitudes 


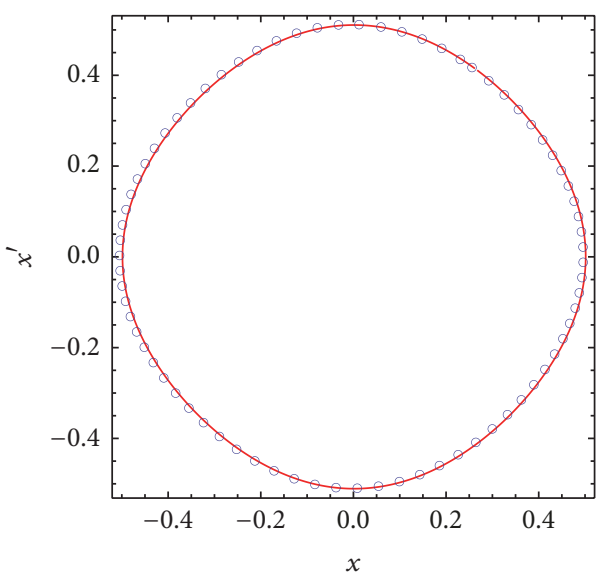

Exact solution

SRHB solution

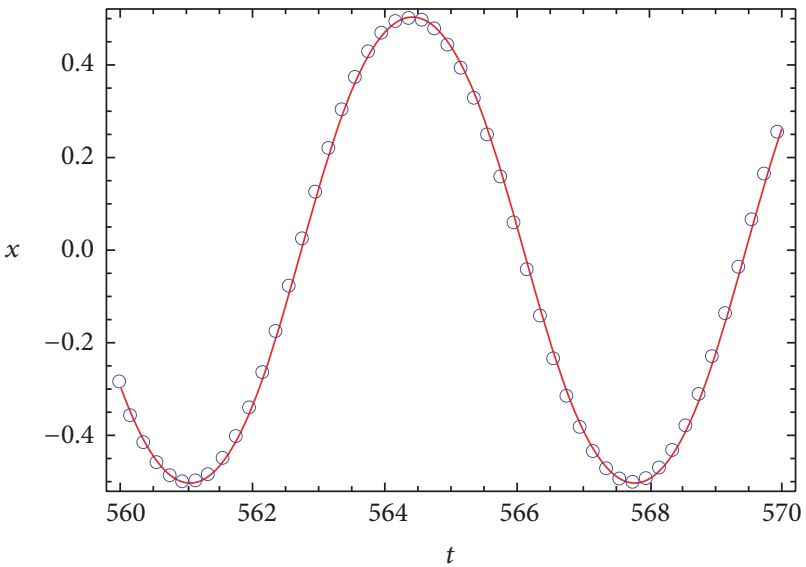

Exact solution

SRHB solution

Figure 3: Comparison of the approximate and exact solutions of Mode 2.

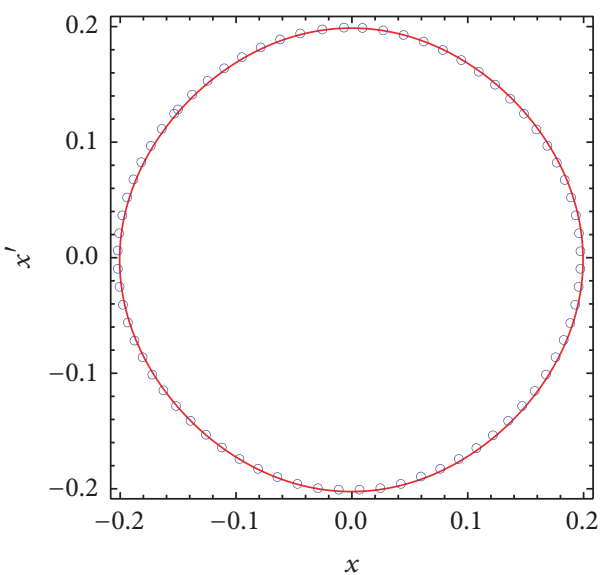

Exact solution SRHB solution

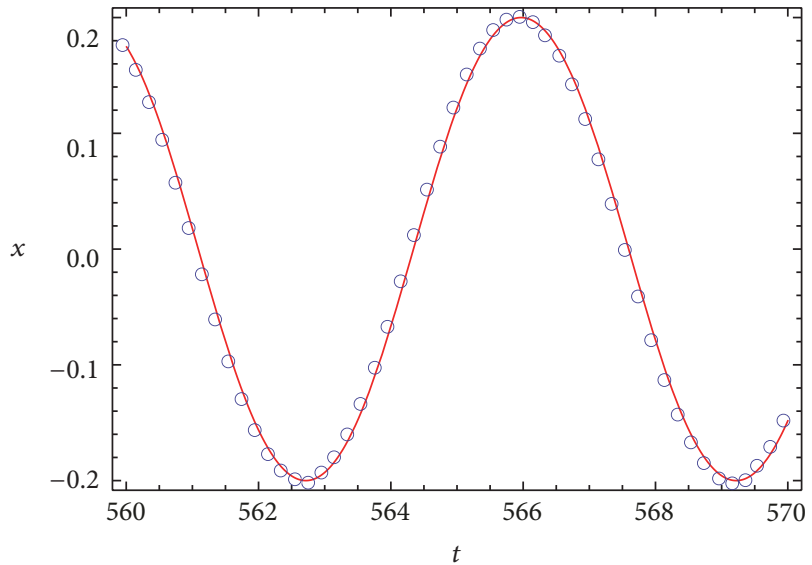

Exact solution

SRHB solution

FIGURE 4: Comparison of the approximate and exact solutions of Mode 3.

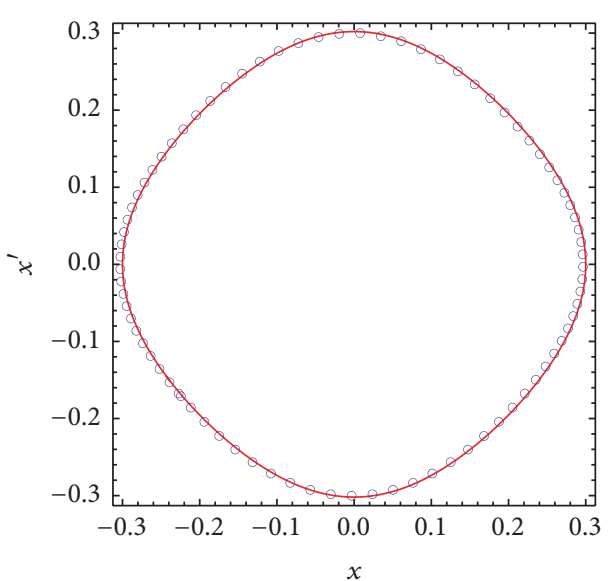

Exact solution

SRHB solution

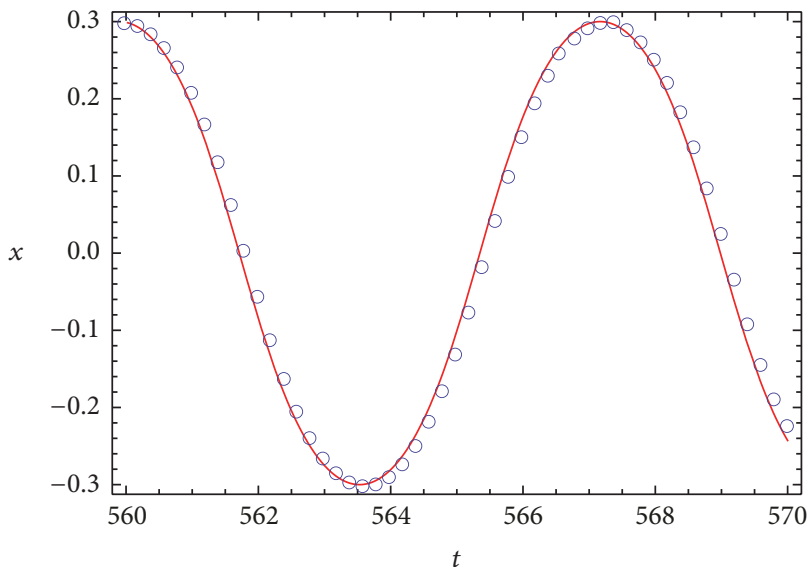

Exact solution

SRHB solution

FIGURE 5: Comparison of the approximate and exact solutions of Mode 4. 
of vibration, it reveals that SRHBM can be used to solve a nonlinear equation with high nonlinearities. According to the figures and tables, it is effective to explain that the presented approximations are more accurate. Therefore, we can conclude that the SRHBM is more available and effective. Ultimately, we consider that the SRHBM can be used to deal with more complex strongly nonlinear vibration problems.

\section{Conflicts of Interest}

The authors declare that there are no conflicts of interest regarding the publication of this paper.

\section{Authors' Contributions}

All the authors contributed equally and significantly to the writing of this paper. All the authors read and approved the final manuscript.

\section{Acknowledgments}

The authors gratefully acknowledge the support of the National Natural Science Foundation of China (NNSFC) through Grants nos. 11572288, 11302184, and 11372015.

\section{References}

[1] R. E. Mickens, Mathematical Methods for the Natural and Engineering Sciences, World Scientific, Singapore, 2004.

[2] O. Abdulaziz, N. F. M. Noor, and I. Hashim, "Homotopy analysis method for fully developed MHD micropolar fluid flow between vertical porous plates," International Journal for Numerical Methods in Engineering, vol. 78, no. 7, pp. 817-827, 2009.

[3] Y. M. Chen and J. K. Liu, "Homotopy analysis method for limit cycle flutter of airfoils," Applied Mathematics and Computation, vol. 203, no. 2, pp. 854-863, 2008.

[4] R. R. Pušenjak, "Extended Lindstedt-Poincare method for non-stationary resonances of dynamical systems with cubic nonlinearities," Journal of Sound \& Vibration, vol. 314, no. 1-2, pp. 194-216, 2008.

[5] P. Amore and A. Aranda, "Improved Lindstedt-Poincaré method for the solution of nonlinear problems," Journal of Sound and Vibration, vol. 283, no. 3-5, pp. 1115-1136, 2005.

[6] M. Senator and C. N. Bapat, "A perturbation technique that works even when the nonlinearity is not small," Journal of Sound and Vibration, vol. 164, no. 1, pp. 1-27, 1993.

[7] Y. K. Cheung, S. H. Chen, and S. L. Lau, "A modified LindstedtPoincaré method for certain strongly nonlinear oscillators," International Journal of Non-Linear Mechanics, vol. 26, no. 3-4, pp. 367-378, 1991.

[8] Q.-Q. Hu, C. W. Lim, and L.-Q. Chen, "Nonlinear vibration of a cantilever with a Derjaguin-Müller-Toporov contact end," International Journal of Structural Stability and Dynamics, vol. 8, no. 1, pp. 25-40, 2008.

[9] K. Huseyin and R. Lin, "An intrinsic multiple-scale harmonic balance method for nonlinear vibration and bifurcation problems," International Journal of Non-Linear Mechanics, vol. 26, no. 5, pp. 727-740, 1991.
[10] L.-L. Ke, J. Yang, S. Kitipornchai, and Y. Xiang, "Flexural vibration and elastic buckling of a cracked timoshenko beam made of functionally graded materials," Mechanics of Advanced Materials and Structures, vol. 16, no. 6, pp. 488-502, 2009.

[11] S. K. Lai, C. W. Lim, B. S. Wu, C. Wang, Q. C. Zeng, and X. F. He, "Newton-harmonic balancing approach for accurate solutions to nonlinear cubic-quintic Duffing oscillators," Applied Mathematical Modelling, vol. 33, no. 2, pp. 852-866, 2009.

[12] S. J. Liao, "An approximate solution technique not depending on small parameters: a special example," International Journal of Non-Linear Mechanics, vol. 30, no. 3, pp. 371-380, 1995.

[13] S. Liao, Beyond Perturbation: Introduction to the Homotopy Analysis Method, vol. 2 of CRC Series: Modern Mechanics and Mathematics, Chapman \& Hall/CRC, Boca Raton, Fla, USA, 2003.

[14] S. J. Liao, "Comparison between the homotopy analysis method and homotopy perturbation method," Applied Mathematics and Computation, vol. 169, no. 2, pp. 1186-1194, 2005.

[15] S. J. Liao, "An optimal homotopy-analysis approach for strongly nonlinear differential equations," Communications in Nonlinear Science and Numerical Simulation, vol. 15, no. 8, pp. 2003-2016, 2010.

[16] C. W. Lim, R. Xu, S. K. Lai, Y. M. Yu, and Q. Yang, "Nonlinear free vibration of an elastically-restrained beam with a point mass via the newton-harmonic balancing approach," International Journal of Nonlinear Sciences and Numerical Simulation, vol. 10, no. 5, pp. 661-674, 2009.

[17] T. Pirbodaghi, S. H. Hoseini, M. T. Ahmadian, and G. H. Farrahi, "Duffing equations with cubic and quintic nonlinearities," Computers and Mathematics with Applications, vol. 57, no. 3, pp. 500-506, 2009.

[18] F. F. Seelig, "Unrestricted harmonic balance II. Application to stiff ordinary differential equations in enzyme catalysis," Journal of Mathematical Biology, vol. 12, no. 2, pp. 187-198, 1981.

[19] J. L. Summers and M. D. Savage, "Two timescale harmonic balance. I. Application to autonomous one-dimensional nonlinear oscillators," Philosophical Transactions of the Royal Society B: Biological Sciences, vol. 340, no. 1659, pp. 473-501, 1992.

[20] R. A. Van Gorder and K. Vajravelu, "On the selection of auxiliary functions, operators, and convergence control parameters in the application of the Homotopy Analysis method to nonlinear differential equations: a general approach," Communications in Nonlinear Science and Numerical Simulation, vol. 14, no. 12, pp. 4078-4089, 2009.

[21] H. Wagner, "Large-amplitude free vibrations of a beam," Journal of Applied Mechanics, vol. 32, no. 4, pp. 887-892, 1965.

[22] C. Wang and I. Pop, "Analysis of the flow of a power-law fluid film on an unsteady stretching surface by means of homotopy analysis method," Journal of Non-Newtonian Fluid Mechanics, vol. 138, no. 2-3, pp. 161-172, 2006.

[23] B. Wu and P. Li, "A method for obtaining approximate analytic periods for a class of nonlinear oscillators," Meccanica, vol. 36, no. 2, pp. 167-176, 2001.

[24] K. Yabushita, M. Yamashita, and K. Tsuboi, "An analytic solution of projectile motion with the quadratic resistance law using the homotopy analysis method," Journal of Physics. A. Mathematical and Theoretical, vol. 40, no. 29, pp. 8403-8416, 2007.

[25] S. S. Ganji, D. D. Ganji, H. Babazadeh, and N. Sadoughi, "Application of amplitude-frequency formulation to nonlinear oscillation system of the motion of a rigid rod rocking back," 
Mathematical Methods in the Applied Sciences, vol. 33, no. 2, pp. 157-166, 2010.

[26] D. D. Ganji, H. B. Rokni, M. G. Sfahani, and S. S. Ganji, "Approximate traveling wave solutions for coupled WhithamBroer-Kaup shallow water," Advances in Engineering Software, vol. 41, no. 7-8, pp. 956-961, 2010.

[27] S. S. Ganji, A. Barari, and D. D. Ganji, "Approximate analysis of two-massspring systems and buckling of a column," Computers and Mathematics with Applications, vol. 61, no. 4, pp. 1088-1095, 2011.

[28] S. S. Ganji, A. Barari, L. B. Ibsen, and G. Domairry, "Differential transform method for mathematical modeling of jamming transition problem in traffic congestion flow," Central European Journal of Operations Research, vol. 20, no. 1, pp. 87-100, 2012.

[29] M. N. Hamdan and N. H. Shabaneh, "On the large amplitude free vibrations of a restrained uniform beam carrying an intermediate lumped mass," Journal of Sound and Vibration, vol. 199, no. 5, pp. 711-736, 1997.

[30] S. Telli and O. Kopmaz, "Free vibrations of a mass grounded by linear and nonlinear springs in series," Journal of Sound and Vibration, vol. 289, no. 4-5, pp. 689-710, 2006.

[31] S. K. Lai and C. W. Lim, "Accurate approximate analytical solutions for nonlinear free vibration of systems with serial linear and nonlinear stiffness," Journal of Sound and Vibration, vol. 307, no. 3, pp. 720-736, 2007.

[32] S.-S. Chen and C.-K. Chen, "Application of the differential transformation method to the free vibrations of strongly nonlinear oscillators," Nonlinear Analysis. Real World Applications, vol. 10, no. 2, pp. 881-888, 2009.

[33] S. S. Ganji, D. D. Ganji, M. G. Sfahani, and S. Karimpour, "Application of AFF and HPM to the systems of strongly nonlinear oscillation," Current Applied Physics, vol. 10, no. 5, pp. 1317-1325, 2010.

[34] I. Mehdipour, D. D. Ganji, and M. Mozaffari, "Application of the energy balance method to nonlinear vibrating equations," Current Applied Physics, vol. 10, no. 1, pp. 104-112, 2010.

[35] Y. H. Qian, S. K. Lai, W. Zhang, and Y. Xiang, "Study on asymptotic analytical solutions using HAM for strongly nonlinear vibrations of a restrained cantilever beam with an intermediate lumped mass," Numerical Algorithms, vol. 58, no. 3, pp. 293-314, 2011.

[36] Z. Guo and W. Zhang, "The spreading residue harmonic balance study on the vibration frequencies of tapered beams," Applied Mathematical Modelling, vol. 40, no. 15, pp. 7195-7203, 2016.

[37] Z. Guo and X. Ma, "Residue harmonic balance solution procedure to nonlinear delay differential systems," Applied Mathematics and Computation, vol. 237, pp. 20-30, 2014.

[38] P. Ju and X. Xue, "Global residue harmonic balance method to periodic solutions of a class of strongly nonlinear oscillators," Applied Mathematical Modelling, vol. 38, no. 24, pp. 6144-6152, 2014.

[39] P. Ju and X. Xue, "Global residue harmonic balance method for large-amplitude oscillations of a nonlinear system," Applied Mathematical Modelling, vol. 39, no. 2, pp. 449-454, 2015.

[40] Y. Y. Lee, "Free vibration analysis of a nonlinear panel coupled with extended cavity using the multi-level residue harmonic balance method," Thin-Walled Structures, vol. 98, pp. 332-336, 2016. 


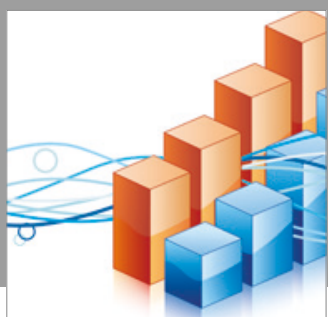

Advances in

Operations Research

vatersals

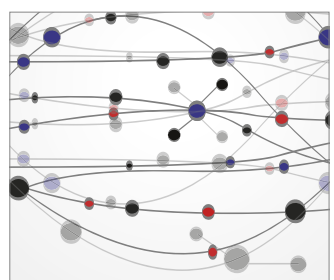

\section{The Scientific} World Journal
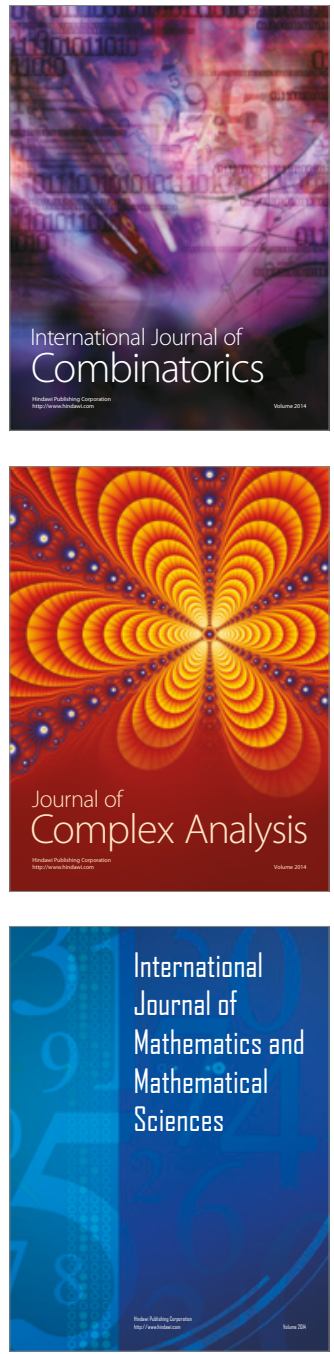
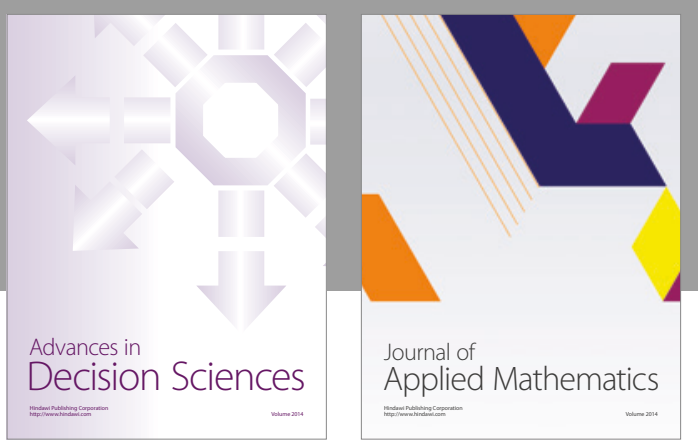

Algebra

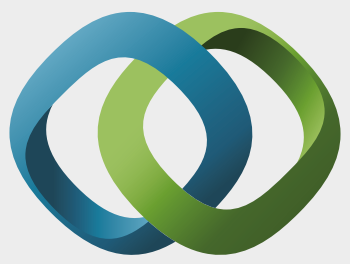

\section{Hindawi}

Submit your manuscripts at

https://www.hindawi.com
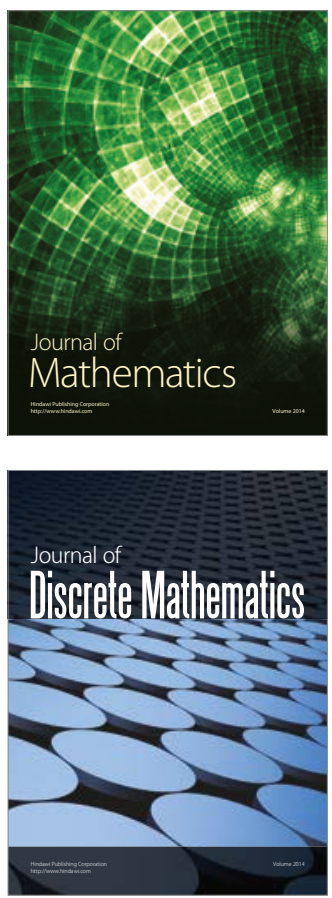

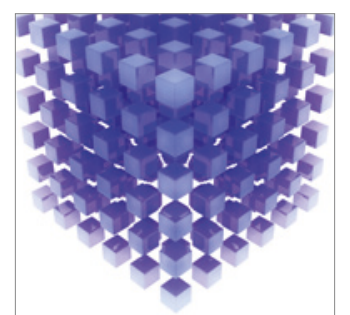

Mathematical Problems in Engineering
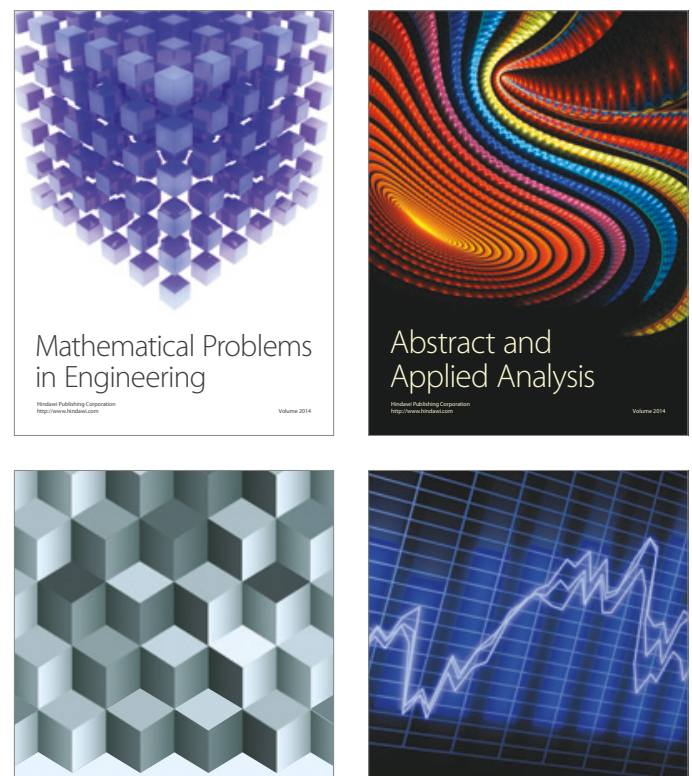

Journal of

Function Spaces

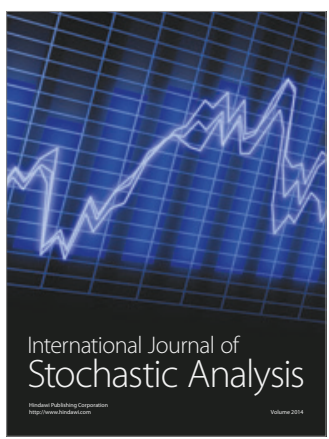

Probability and Statistics
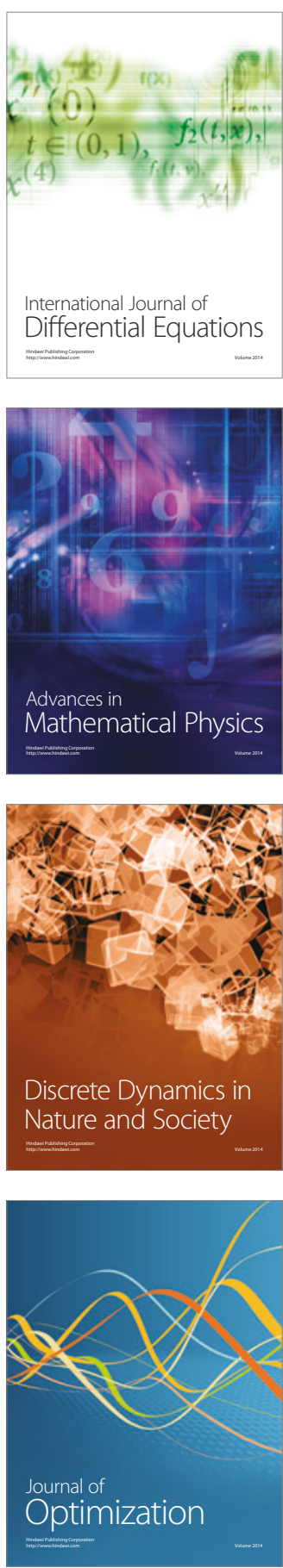